\title{
The Akhmediev breather is unstable
}

\author{
Miguel A. Alejo ${ }^{1}$ Luca Fanelli² Claudio Muñoz $^{3}$ (1) \\ Published online: 26 August 2019 \\ (C) Instituto de Matemática e Estatística da Universidade de São Paulo 2019
}

\section{Abstract}

In this note, we give a rigorous proof that the NLS periodic Akhmediev breather is unstable. The proof follows the ideas in Muñoz (Proyecciones (Antofagasta) 36(4):653-683, 2017), in the sense that a suitable modification of the Stokes wave is the global attractor of the local Akhmediev dynamics for sufficiently large time, and therefore the latter cannot be stable in any suitable finite energy periodic Sobolev space.

Keywords Akhmediev $\cdot$ Breather $\cdot$ Stability $\cdot$ Schrödinger

Mathematics Subject Classification 35Q55 · 35Q51; 35Q35 · 35Q40

M. A. A. also would like to thank to the Departamento de Ingeniería Matemática (DIM) of U. Chile, where part of this work was completed, for its kind hospitality and support. Funded by Product. CNPq Grant No. 305205/2016-1 and MathAmSud/Capes EEQUADD collaboration Math16-01.

C. M. was partly funded by Chilean research Grants FONDECYT 1150202, and CMM Conicyt PIA AFB170001.

The authors were participants of the Third Workshop on Nonlinear Dispersive Equations, held in Campinas, Brazil, during November 8-10 2017. They acknowledge the support and charming ambiance ensured by UNICAMP and the organizers of this event.

$\bowtie$ Claudio Muñoz

cmunoz@dim.uchile.cl

Miguel A. Alejo

miguel.alejo@ufsc.br

Luca Fanelli

fanelli@mat.uniroma1.it

1 Universidade Federal de Santa Catarina, Florianópolis, Brasil

2 Dipartimento di Matematica, SAPIENZA Università di Roma, P. le Aldo Moro 5, 00185 Rome, Italy

3 CNRS and Departamento de Ingeniería Matemática and Centro de Modelamiento Matemático (UMI 2807 CNRS), Universidad de Chile, Casilla 170 Correo 3, Santiago, Chile 


\section{Introduction}

Let $a \in\left(0, \frac{1}{2}\right)$. The Akhmediev breather [2]

$$
\begin{aligned}
A(t, x) & :=e^{i t}\left[1+\frac{\alpha^{2} \cosh (\beta t)+i \beta \sinh (\beta t)}{\sqrt{2 a} \cos (\alpha x)-\cosh (\beta t)}\right], \quad t, x \in \mathbb{R}, \\
\beta & =(8 a(1-2 a))^{1 / 2}, \quad \alpha=(2(1-2 a))^{1 / 2},
\end{aligned}
$$

is a $\frac{2 \pi}{a}$-periodic in space, localized in time smooth solution to the focusing cubic nonlinear Schrödinger equation (NLS) in one dimension:

$$
i \partial_{t} u+\partial_{x}^{2} u+|u|^{2} u=0, \quad u=u(t, x) \in \mathbb{C}, \quad t, x \in \mathbb{R} .
$$

See Figs. 1 and 2 for details. This equation appears as a model of propagation of light in nonlinear optical fibers (with different meanings for time and space variables), as well as in small-amplitude gravity waves on the surface of deep inviscid water. Additionally, this equation is completely integrable, as showed by Zakharov and Shabat [20].

A particular feature of $A$ above is its nonzero boundary value at infinity in time and space. Indeed, $A$ converges, as $t \rightarrow \pm \infty$, to the Stokes wave $e^{i t}$, also solution of (1.2):

$$
\lim _{t \rightarrow \pm \infty}\left\|A(t, x)-e^{ \pm i \theta} e^{i t}\right\|_{H_{\sharp}^{1}}=0, \quad e^{i \theta}=1-\alpha^{2}-i \beta .
$$

Here, $H_{\sharp}^{S}:=H_{\sharp}^{S}\left(\left(0, \frac{2 \pi}{a}\right)\right)$ denotes the standard Sobolev space $H^{s}$ of $\frac{2 \pi}{a}$-space periodic functions. Consequently, $A(t, x)$ exemplifies the modulational instability phenomenon, which - roughly speaking — says that small perturbations of the Stokes wave are unstable and grow quickly. This unstable growth leads to a nontrivial com-

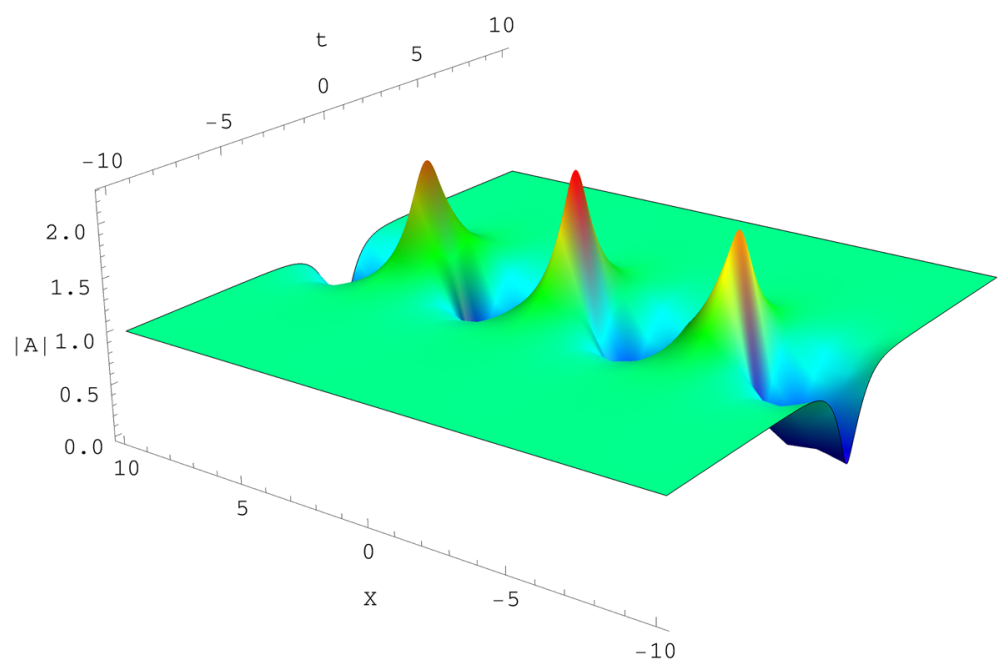

Fig. $1|A|$ with $a=0.2$ 


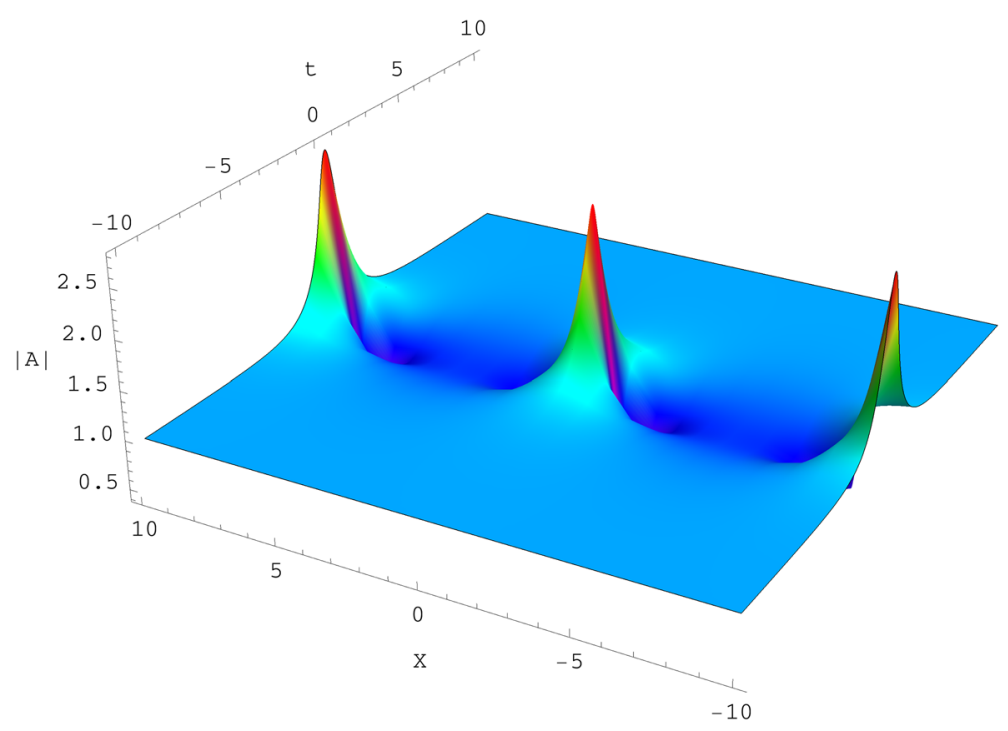

Fig. $2|A|$ with $a=0.4$

petition with the (focusing) nonlinearity, time at which the solution is apparently stabilized. The Akhmediev breather is also a candidate to explain the famous rogue waves. An alternative explanation to the rogue wave phenomena is given by the notion of dispersive blow-up, see Bona and Saut [10].

Two standard conserved quantities for (1.2) in the periodic setting are

$$
M[u]:=\int_{0}^{\frac{2 \pi}{a}}\left(|u|^{2}-1\right), \quad \text { (Mass) }
$$

and

$$
E[u]:=\int_{0}^{\frac{2 \pi}{a}}\left(\left|u_{x}\right|^{2}-\frac{1}{2}\left(|u|^{2}-1\right)^{2}\right), \quad \text { (Energy) } .
$$

A third one is given by [5]

$$
F[u]:=\int_{0}^{\frac{2 \pi}{a}}\left(\left|u_{x x}\right|^{2}-3\left(|u|^{2}-1\right)\left|u_{x}\right|^{2}-\frac{1}{2}\left(\left(|u|^{2}\right)_{x}\right)^{2}+\frac{1}{2}\left(|u|^{2}-1\right)^{3}\right) .
$$

This third conserved quantity appears from the integrability of the equation.

In this paper, we continue the work started by one of us in [17], where we proved that the Peregrine $[1,19]$ and Kuznetsov-Ma $[13,15]$ breathers are unstable under finite energy perturbations in any Sobolev space $H^{s}(\mathbb{R}), s>\frac{1}{2}$. Previously, the Peregrine soliton was showed to be numerically unstable under small perturbations by Klein and Haragus [12]. See [17] for more details on those breathers, as well as a more or less accurate account of the current literature. 
However, the stability analysis of (1.1) was left open because of its spatial periodic behavior. Our first and main result is the following:

Theorem 1.1 The Akhmediev breather (1.1) is unstable under small perturbations in $H_{\sharp}^{S}, s>\frac{1}{2}$.

By stability, we mean the following concept [17]. Fix $s>\frac{1}{2}$, and $t_{0} \in \mathbb{R}$. We say that a particular $\frac{2 \pi}{a}$-periodic globally defined solution $U=e^{i t}(1+W)$ of (1.2) is orbitally stable in $H_{\sharp}^{S}\left(\frac{2 \pi}{a}\right)$ if there are constants $C_{0}, \varepsilon_{0}>0$ such that, for any $0<\varepsilon<\varepsilon_{0}$,

$$
\begin{gathered}
\left\|u_{0}-U\left(t_{0}\right)\right\|_{H_{\sharp}^{s}}<\varepsilon \\
\Downarrow \\
\exists x_{0}(t), \gamma_{0}(t) \in \mathbb{R} \text { such that } \sup _{t \in \mathbb{R}}\left\|u(t)-e^{i \gamma(t)} U\left(t, x-x_{0}(t)\right)\right\|_{H_{\sharp}^{s}}<C_{0} \varepsilon .
\end{gathered}
$$

Here $u(t)$ is the solution to the IVP (1.2) with initial datum $u\left(t_{0}\right)=u_{0}$ (see Proposition 2.1), and $x_{0}(t), \gamma_{0}(t)$ can be assumed continuous because the IVP is well-posed in a continuous-in-time Sobolev space. If (1.7) is not satisfied, we will say that $U$ is unstable. Note additionally that condition (1.7) requires $w$ globally defined, otherwise $U$ is trivially unstable, since $U$ is globally defined.

The proof of Theorem 1.1 uses (1.3) in a crucial way: a modified Stokes wave is an attractor of the dynamics around the Akhmediev breather for large time. See also [8,9] for numerical studies of the stability of $\mathrm{mKdV}$ and Sine-Gordon breathers in the periodic and nonperiodic settings. Other rigorous stability results for breathers can be found in $[3,6,7,16,18]$.

No NLS (1.2) breather seems to be stable. In fact, Peregrine, Kuznetsov-Ma and now Akhmediev were shown to be unstable. This is not necessarily consequence of the nonzero background. Indeed, even breathers on zero background [5], called SatsumaYajima breathers, are unstable.

Being $A$ unstable, it does not mean that it has no structure at all. In this paper we advance, following the ideas introduced in [5], that indeed, $A$ has a very rich (unstable) variational structure. In particular,

Theorem 1.2 The Akhmediev breather A (1.1) is a critical point of the functional

$$
\mathcal{H}[u]:=F[u]-\alpha^{2} E[u],
$$

that is to say, $\mathcal{H}^{\prime}[A][w]=0$ for all $w \in H_{\sharp}^{2}$. In particular, for each $t \in \mathbb{R} A(t, x)$ satisfies the nonlinear $O D E$

$$
\begin{gathered}
A_{(4 x)}+3 A_{x}^{2} \bar{A}+\left(4|A|^{2}-3\right) A_{x x}+A^{2} \bar{A}_{x x}+2\left|A_{x}\right|^{2} A \\
+\frac{3}{2}\left(|A|^{2}-1\right)^{2} A+\alpha^{2}\left(A_{x x}+\left(|A|^{2}-1\right) A\right)=0 .
\end{gathered}
$$


The proof of this result follows easily from the methods in [5], in which one expands $\mathcal{H}[A+w]$. We get

$$
\mathcal{H}[A+w]=\mathcal{H}[A]+\mathcal{H}^{\prime}[A][w]+O\left(\|w\|_{H_{\sharp}^{2}}^{2}\right) .
$$

Then, performing some lengthy computations, one proves that $\mathcal{H}^{\prime}[A][w]=0$ independently of $w$. See Sect. 3 for the proof.

We believe that the variational structure appearing in breather solutions is independent of the well-posed character of the equation. In particular, we claim that the explicit breather of the strongly ill-posed bad Boussinesq equation

$$
u_{t t}-u_{x x}-\left(u_{x x}+\frac{3}{2} u^{2}\right)_{x x}=0
$$

has an associated rich variational structure [4].

\section{Proof of Theorem 1.1}

The proof is not difficult at all. We just need a preliminary well-posedness result. Set

$$
u(t, x)=A(t, x)+w(t, x), \quad w \text { unknown. }
$$

Then (1.2) becomes a modified NLS equation

$$
\begin{aligned}
i \partial_{t} w+\partial_{x}^{2} w & =-G[w], \\
G[w] & :=|A+w|^{2}(A+w)-|A|^{2} A .
\end{aligned}
$$

Proposition 2.1 The NLS Eq.(1.2) is locally well-posed for any initial data at time $t=t_{0}$ of the form $A\left(t_{0}, x\right)+w_{0}(x)$, with $w_{0} \in H_{\sharp}^{s}, s>\frac{1}{2}$.

Proof See “Appendix B".

Note that there is always a local solution $u$ of (1.2) such that $u(t)=A(t)+w(t)$, with $w \in H_{\sharp}^{s}$. In particular, given time dependent parameters $x_{0}(t), \gamma_{0}(t) \in \mathbb{R}$, if the decomposition $u(t)=e^{i \gamma_{0}(t)} A\left(t, x-x_{0}(t)\right)+\tilde{w}(t)$ holds, then $\tilde{w}(t)$ still belongs to $H_{\sharp}^{S}$. This is not true in the non periodic case, see [17].

We did not try to improve the local well-posedness result for (2.2) because the flow contains a non oscillatory bad component in the case of small frequencies, see [17] for details. In particular, Strichartz estimates are not available in this case. Also, the global well-posedness of (2.2) is an open question. 


\subsection{End of proof}

We only treat the case $t \rightarrow+\infty$, the other being very similar. Fix $s>\frac{1}{2}$. Let us assume that the Akhmediev breather $A$ in (1.1) is orbitally stable, as in (1.7). Write (see (1.3))

$$
\begin{aligned}
& A(t, x)=e^{i t}\left(e^{i \theta}+Q(t, x)\right), \\
& Q(t, x):=\frac{\alpha^{2} \cosh (\beta t)+i \beta \sinh (\beta t)}{\sqrt{2 a} \cos (\alpha x)-\cosh (\beta t)}+\left(\alpha^{2}+i \beta\right) .
\end{aligned}
$$

Now consider, as a perturbation of the Akhmediev breather, the $\frac{2 \pi}{a}$-periodic Stokes wave $e^{i \theta} e^{i t}$. Indeed, we have [see (2.3)],

$$
\lim _{t \rightarrow+\infty}\left\|A(t)-e^{i \theta} e^{i t}\right\|_{H_{\sharp}^{s}}=\lim _{t \rightarrow+\infty}\|Q(t)\|_{H_{\sharp}^{s}}=0 .
$$

Indeed, this follows from the identity

$$
Q(t, x)=\alpha^{2}\left(1-\frac{1}{1-\sqrt{2 a} \frac{\cos (\alpha x)}{\cosh (\beta t)}}\right)+i \beta\left(1-\frac{\tanh (\beta t)}{1-\sqrt{2 a} \frac{\cos (\alpha x)}{\cosh (\beta t)}}\right) .
$$

Therefore, we have two solutions to (1.2) that converge to the same profile as $t \rightarrow+\infty$. This fact contradicts the orbital stability, since for $x_{0}(t), \gamma_{0}(t) \in \mathbb{R}$ given in (1.7), and the definition of $A$ in (1.1),

$$
\begin{aligned}
& \left\|e^{i \theta}-e^{i \gamma_{0}(0)} A\left(0, x-x_{0}(0)\right)\right\|_{H_{\sharp}^{s}} \\
& \quad=\left\|e^{i \theta}-e^{i \gamma_{0}(0)}\left[1+\frac{\alpha^{2}}{\sqrt{2 a} \cos \left(\alpha\left(x-x_{0}(0)\right)-1\right.}\right]\right\|_{H_{\sharp}^{s}} \\
& \quad=c_{s}>0,
\end{aligned}
$$

is a fixed number, but if $t_{0}=T$ is taken large enough, $\|Q(T)\|_{H_{\sharp}^{s}}$ can be made arbitrarily small. Indeed, by classical interpolation $\left(\|u\|_{H_{\sharp}^{s}}^{2}:=\sum_{n \geq 0} n^{2 s}|\hat{u}(n)|^{2}\right.$, and $n^{2 s}=n^{2(0+s .1)}$ and Hölder)

$$
\|Q(T)\|_{H_{\sharp}^{s}} \lesssim s\|Q(T)\|_{L_{\sharp}^{2}}^{1-s}\|Q(T)\|_{H_{\sharp}^{1}}^{s}, \quad s \in(0,1) .
$$

Now, to evaluate $\lim _{t \rightarrow+\infty}\|Q(t)\|_{L_{\sharp}^{2}}$ requires some care. Clearly from (2.4) we have $Q(t, x) \rightarrow 0$ as $t \rightarrow+\infty$, for all $x \in\left[0, \frac{2 \pi}{\alpha}\right)$. Also,

$$
|Q(t, x)| \lesssim \frac{\alpha^{2} \sqrt{2 a}}{(1-\sqrt{2 a}) \cosh (\beta t)}+\frac{\beta}{(1-\sqrt{2 a})}\left((1-\tanh (\beta t))+\frac{\sqrt{2 a}}{\cosh (\beta t)}\right) .
$$


Therefore, by using dominated convergence we conclude. As for the derivative, note that

$$
\partial_{x} Q(t, x)=\frac{\alpha^{3} \sqrt{2 a} \sin (\alpha x)}{\cosh (\beta t)\left(1-\sqrt{2 a} \frac{\cos (\alpha x)}{\cosh (\beta t)}\right)^{2}}+i \frac{\alpha \beta \sqrt{2 a} \tanh (\beta t) \sin (\alpha x)}{\left(1-\sqrt{2 a} \frac{\cos (\alpha x)}{\cosh (\beta t)}\right)^{2}} .
$$

Proceeding in a similar fashion as in the $L^{2}$ norm, we have $\lim _{t \rightarrow+\infty}\left\|\partial_{x} Q(t)\right\|_{L_{\sharp}^{2}}=0$. Therefore, we conclude from (2.5) that $\|Q(T)\|_{H_{\sharp}^{s}}$ can be made arbitrarily small if $T$ is sufficiently large.

Note finally that the Cauchy problem for (1.2) with initial data at time $T$ given by $u_{0}=e^{i T} e^{i \theta}=A(T)-e^{i T} Q(T)$ is well-defined from Proposition 2.1, since $e^{i T} Q(T) \in H_{\sharp}^{S}$. This proves Theorem 1.1.

Remark 2.1 We conjecture that any soliton solution constructed using Bäcklund transformations, with attached Akhmediev breathers, must be unstable.

\section{Proof of Theorem 1.2}

Explicitly, we have from (1.5) and (1.6), integration by parts, and the periodic character of $A$ and its spatial derivatives at the boundaries, and $w$ its first and second spatial derivatives,

$$
\begin{aligned}
& \mathcal{H}[A+w]=F[A+w]-\alpha^{2} E[A+w] \\
& =\int_{0}^{\frac{2 \pi}{a}}\left(\left|A_{x x}+w_{x x}\right|^{2}-3\left(|A+w|^{2}-1\right)\left|A_{x}+w_{x}\right|^{2}-\frac{1}{2}\left(\left(|A+w|^{2}\right)_{x}\right)^{2}\right. \\
& \left.+\frac{1}{2}\left(|A+w|^{2}-1\right)^{3}\right) \\
& -\alpha^{2} \int_{0}^{\frac{2 \pi}{a}}\left(\left|A_{x}+w_{x}\right|^{2}-\frac{1}{2}\left(|A+w|^{2}-1\right)^{2}\right) \\
& =\mathcal{H}[A]+\int_{0}^{\frac{2 \pi}{a}}\left(2 \operatorname{Re}\left(A_{4 x} \bar{w}\right)-3\left(|A|^{2}-1\right) 2 \operatorname{Re}\left(A_{x} \bar{w}_{x}\right)\right. \\
& -3(2 \operatorname{Re}(A \bar{w}))\left|A_{x}\right|^{2} \\
& \left.-\left(|A|^{2}\right)_{x}(2 \operatorname{Re}(A \bar{w}))_{x}+\frac{3}{2}\left(|A|^{2}-1\right)^{2} 2 \operatorname{Re}(A \bar{w})\right) \\
& -\alpha^{2} \int_{0}^{\frac{2 \pi}{a}}\left(-2 \operatorname{Re}\left(A_{x x} \bar{w}\right)-\left(|A|^{2}-1\right) 2 \operatorname{Re}(A \bar{w})\right)+O\left(\|w\|_{H_{\sharp}^{2}}^{2}\right) \\
& =\mathcal{H}[A]+2 \operatorname{Re} \int_{0}^{\frac{2 \pi}{a}}\left(A_{4 x} \bar{w}\right. \\
& -3\left(|A|^{2}-1\right) A_{x} \bar{w}_{x}-3 A \bar{w}\left|A_{x}\right|^{2}+\left(|A|^{2}\right)_{x x} A \bar{w} \\
& \left.+\frac{3}{2}\left(|A|^{2}-1\right)^{2} A \bar{w}-\alpha^{2}\left[-A_{x x} \bar{w}-\left(|A|^{2}-1\right) A \bar{w}\right]\right)+O\left(\|w\|_{H_{\sharp}^{2}}^{2}\right)
\end{aligned}
$$




$$
\begin{aligned}
= & \mathcal{H}[A] \\
& +2 \operatorname{Re} \int_{0}^{\frac{2 \pi}{a}} \bar{w}\left(A_{4 x}+3\left(|A|^{2}-1\right) A_{x x}+3\left(A_{x}^{2} \bar{A}+A\left|A_{x}\right|^{2}\right)-3 A\left|A_{x}\right|^{2}\right. \\
& +A_{x x}|A|^{2}+A^{2} \bar{A}_{x x}+2 A\left|A_{x}\right|^{2}+\frac{3}{2}\left(|A|^{2}-1\right)^{2} A \\
& \left.+\alpha^{2}\left(A_{x x}+\left(|A|^{2}-1\right) A\right)\right)+O\left(\|w\|_{H_{\sharp}^{2}}^{2}\right) \\
= & \mathcal{H}[A]+2 \operatorname{Re} \int_{0}^{\frac{2 \pi}{a}} \bar{w}\left(A_{(4 x)}+3 A_{x}^{2} \bar{A}+\left(4|A|^{2}-3\right) A_{x x}\right. \\
& +A^{2} \bar{A}_{x x}+2\left|A_{x}\right|^{2} A \\
& \left.+\frac{3}{2}\left(|A|^{2}-1\right)^{2} A+\alpha^{2}\left(A_{x x}+\left(|A|^{2}-1\right) A\right)\right)+O\left(\|w\|_{H_{\sharp}^{2}}^{2}\right),
\end{aligned}
$$

and therefore we get

$$
\mathcal{H}[A+w]=\mathcal{H}[A]+\mathcal{H}^{\prime}[A][w]+O\left(\|w\|_{H_{\sharp}^{2}}^{2}\right) .
$$

Then, performing some lengthy computations (see "Appendix A") one proves that $\mathcal{H}^{\prime}[A][w]=0$ independently of $w$. This proves Theorem 1.2.

Acknowledgements We thank the referee for his/her fruitful comments and suggestions which helped to improve this paper.

\section{Compliance with ethical standards}

Conflicts of interest The authors certify that no conflict of interest, of any possible type, is affected to this article.

\section{Appendix A: Proof of (1.8)}

Following [5], let us use the notation for the Akhmediev breather solution (1.1):

$$
\begin{aligned}
A & =e^{i t}\left(1+\frac{M}{N}\right), \quad \text { with } \\
M & :=\alpha^{2} \cosh (\beta t)+i \beta \sinh (\beta t), \\
N & :=\sqrt{2 a} \cos (\alpha x)-\cosh (\beta t) .
\end{aligned}
$$

Now, we rewrite the identity (1.8) in terms of $M, N$ in the following way

$$
\text { (1.8) }=\frac{e^{i t}}{N^{5}} \sum_{i=1}^{6} R_{i}
$$


with $R_{i}$ given explicitly by:

$$
\begin{aligned}
R_{1}:= & \frac{1}{2} N\left(6 i M N_{t} N_{x}^{2}-2 i N\left(N_{x}\left(M_{t} N_{x}+M\left(i N_{x}+2 N_{x t}\right)\right)+N_{t}\left(2 M_{x} N_{x}+M N_{x x}\right)\right)\right. \\
& +N^{3}\left(M_{x x}-i M_{x x t}\right)+N^{2}\left(-2 M_{x}\left(N_{x}-i N_{x t}\right)\right. \\
& \left.\left.+i\left(2 N_{x} M_{x t}+N_{t} M_{x x}+i M N_{x x}+M_{t} N_{x x}+M N_{x x t}\right)\right)\right) \\
R_{2}:= & \frac{1}{2}\left(2 M(\bar{M}+N)+N\left(2 \bar{M}+\left(\alpha^{2}-1\right) N\right)\right) \\
& \cdot\left(2 M N_{x}^{2}+N^{2} M_{x x}-N\left(2 M_{x} N_{x}+M N_{x x}\right)\right) \\
R_{3}:= & (M+N)\left(-N M_{x}+M N_{x}\right)\left(N \bar{M}_{x}-\bar{M} N_{x}\right) \\
R_{4}:= & \frac{1}{2}(\bar{M}+N)\left(N M_{x}-M N_{x}\right)^{2} \\
R_{5}:= & \frac{1}{2} N^{2}(M+N)\left(\left(\frac{3}{2}-\alpha^{2}\right) N^{2}+\left(-3+\alpha^{2}\right)(\bar{M}+N)(M+N)\right) \\
\text { and } & \\
R_{6}: & =\frac{3}{4}(M+N)^{3}(\bar{M}+N)^{2} .
\end{aligned}
$$

Now substituting the explicit functions $M, N$ (A.1) in $R_{i}, i=1, \ldots, 6$ and collecting terms, we get

$$
\begin{aligned}
\sum_{i=1}^{6} R_{i}= & a_{1} \cosh (t \beta)+a_{2} \cosh ^{3}(\beta t)+a_{3} \cosh ^{5}(\beta t)+a_{4} \sinh (t \beta) \\
& +a_{5} \cosh ^{2}(\beta t) \sinh (\beta t) \\
& +a_{6} \cosh ^{4}(\beta t) \sinh (\beta t)+a_{7} \cos (\alpha x)+a_{8} \cosh ^{2}(\beta t) \cos (\alpha t) \\
& +a_{9} \cosh ^{4}(\beta t) \cos (\alpha x) \\
& +a_{10} \cosh (\beta t) \sinh (\beta t) \cos (\alpha x)+a_{11} \cosh ^{3}(\beta t) \sinh (\beta t) \cos (\alpha x) \\
& +a_{12} \cosh (\beta t) \cos ^{2}(\alpha x) \\
& +a_{13} \cosh ^{3}(\beta t) \cos ^{2}(\alpha x)+a_{14} \cosh ^{2}(\beta t) \cos ^{3}(\alpha x)+a_{15} \cosh (\beta t) \cos ^{4}(\alpha x),
\end{aligned}
$$

with coefficients $a_{i}, i=1, \ldots, 15$ given as follows

$$
\begin{aligned}
& a_{1}=\frac{3}{2}\left(-1+\alpha^{2}\right) \beta^{2}\left(-4 a \alpha^{2}+\beta^{2}\right), \\
& a_{2}=\left(-\left(-1+\alpha^{2}\right) \beta^{2}\left(-5 \alpha^{2}+3 \alpha^{4}+3 \beta^{2}\right)+2 a\left(-5 \alpha^{6}+3 \alpha^{8}-\alpha^{2} \beta^{2}+3 \alpha^{4} \beta^{2}\right)\right), \\
& a_{3}=\frac{1}{2}\left(-1+\alpha^{2}\right)\left(-10 \alpha^{6}+3 \alpha^{8}-10 \alpha^{2} \beta^{2}+3 \beta^{4}+\alpha^{4}\left(8+6 \beta^{2}\right)\right), \\
& a_{4}=\frac{3}{2} i \beta^{3}\left(-4 a \alpha^{2}+\beta^{2}\right), \\
& a_{5}=i \beta\left(\beta^{2}\left(5 \alpha^{2}-3 \alpha^{4}-3 \beta^{2}\right)+a\left(-8 \alpha^{4}+6 \alpha^{6}+6 \alpha^{2} \beta^{2}\right)\right), \\
& a_{6}=\frac{1}{2} i \beta\left(-10 \alpha^{6}+3 \alpha^{8}-10 \alpha^{2} \beta^{2}+3 \beta^{4}+\alpha^{4}\left(8+6 \beta^{2}\right)\right),
\end{aligned}
$$




$$
\begin{aligned}
a_{7} & =\frac{3}{2} \sqrt{2 a} \beta^{2}\left(-4 a \alpha^{2}+\beta^{2}\right), \\
a_{8} & =-\sqrt{2 a}\left(\beta^{2}\left(-7 \alpha^{2}+5 \alpha^{4}+3 \beta^{2}\right)+a\left(-6 \alpha^{6}+2 \alpha^{2} \beta^{2}\right)\right), \\
a_{9} & =\frac{1}{2} \sqrt{2 a}\left(-24 \alpha^{6}+7 \alpha^{8}-16 \alpha^{2} \beta^{2}+3 \beta^{4}+10 \alpha^{4}\left(2+\beta^{2}\right)\right), \\
a_{10} & =2 i \sqrt{2 a} \alpha^{2} \beta\left(4 a \alpha^{2}-\beta^{2}\right), \\
a_{11} & =2 i \sqrt{2 a} \alpha^{2} \beta\left(-2 \alpha^{2}+\alpha^{4}+\beta^{2}\right), \\
a_{12} & =4 a \alpha^{2}\left(-\beta^{2}+2 a\left(\alpha^{4}+\beta^{2}\right)\right), \\
a_{13} & =6 a \alpha^{2}\left(-2 \alpha^{2}+\alpha^{4}+\beta^{2}\right), \\
a_{14} & =2 \sqrt{2 a} a \alpha^{2}\left(-2 \alpha^{2}+\alpha^{4}+\beta^{2}\right), \\
a_{15} & =-4 a^{2} \alpha^{2}\left(-2 \alpha^{2}+\alpha^{4}+\beta^{2}\right) .
\end{aligned}
$$

Finally, using that $\alpha=\sqrt{2(1-2 a)}$ and $\beta=\sqrt{8 a(1-2 a)}$, we have that all $a_{i}$ vanish, and we conclude.

\section{Appendix B: Sketch of Proof of Proposition 2.1}

First of all, we have from (2.2) that

$$
G[w]=2 A \operatorname{Re}(A \bar{w})+|A|^{2} w+A|w|^{2}+2 \operatorname{Re}(A \bar{w}) w+|w|^{2} w .
$$

By scaling and the subcritical character of (2.2), we can assume that the linear term in $G[w]$ above is small. We can also assume the initial time $t_{0}=0$. By the Duhamel's formula, we have

$$
w(t)=e^{i t \partial_{x}^{2}} w_{0}-\int_{0}^{t} e^{i(t-s) \partial_{x}^{2}} G[w](s) d s
$$

Hence, applying the standard Sobolev estimates in $H_{\sharp}^{S}$, with $s>\frac{1}{2}$, we readily obtain the contraction principle required. Note that no use of Strichartz estimates is needed. See [11] or [14] for additional details on the fixed point argument. We skip the details.

\section{References}

1. Akhmediev, N., Ankiewicz, A., Taki, M.: Waves that appear from nowhere and disappear without a trace. Phys. Lett. A 373, 675-678 (2009)

2. Akhmediev, N., Korneev, V.I.: Modulation instability and periodic solutions of the nonlinear Schrödinger equation. Theor. Math. Phys. 69, 1089-1093 (1986)

3. Alejo, M.A.: Nonlinear stability of Gardner breathers. J. Diff. Equ. 264(2), 1192-1230 (2018)

4. Alejo, M.A., Cortez, F., Muñoz, C.: Variational structure of breathers in strongly ill-posed equations (2018) (under preparation)

5. Alejo, M.A., Fanelli, L., Muñoz, C.: Stability and instability of breathers in the $U(1)$ Sasa-Satsuma and nonlinear Schrödinger models. Preprint arXiv:1901.10381

6. Alejo, M.A., Muñoz, C.: Nonlinear stability of mKdV breathers. Commun. Math. Phys. 324(1), 233$262(2013)$ 
7. Alejo, M.A., Muñoz, C.: Dynamics of complex-valued modified KdV solitons with applications to the stability of breathers. Anal. PDE 8(3), 629-674 (2015)

8. Alejo, M.A., Muñoz, C., Palacios, J.M.: On the variational structure of breather solutions I: SineGordon equation. J. Math. Anal. Appl. 453(2), 1111-1138 (2017)

9. Alejo, M.A., Muñoz, C., Palacios, J.M.: On the variational structure of breather solutions II: periodic mKdV equation. EJDE 2017(56), 1-26 (2017)

10. Bona, J.L., Saut, J.-C.: Dispersive blowup of solutions of generalized Korteweg-de Vries equations. J. Differ. Equ. 103(1), 3-57 (1993)

11. Cazenave, T.: Semilinear Schrödinger Equations, Courant Lecture Notes in Mathematics, vol. 10. New York University, Courant Institute of Mathematical Sciences, New York; American Mathematical Society, Providence (2003). ISBN 0-8218-3399-5

12. Klein, C., Haragus, M.: Numerical study of the stability of the Peregrine breather. Ann. Math. Sci. Appl. 2(2), 217-239 (2017). Preprint arXiv:1507.06766

13. Kuznetsov, E.: Solitons in a parametrically unstable plasma. Sov. Phys. Dokl. 22, 507-508 (1977)

14. Linares, F., Ponce, G.: Introduction to Nonlinear Dispersive Equations. Universitext, 2nd edn. Springer, New York (2015)

15. Ma, Y.C.: The perturbed plane-wave solutions of the cubic Schrödinger equation. Stud. Appl. Math. 60, 43-58 (1979)

16. Muñoz, C.: Stability of integrable and nonintegrable structures. Adv. Differ. Equ. 19(9-10), 947-996 (2014)

17. Muñoz, C.: Instability in nonlinear Schrödinger breathers. Proyecciones (Antofagasta) 36(4), 653-683 (2017)

18. Muñoz, C., Palacios, J.M.: Nonlinear stability of 2-solitons of the Sine-Gordon equation in the energy space. Ann. IHP Anal. Nonlinéaire 36(4), 977-1034 (2019)

19. Peregrine, D.H.: Water waves, nonlinear Schrödinger equations and their solutions. J. Aust. Math. Soc. Ser. B 25, 16-43 (1983)

20. Zakharov, V.E., Shabat, A.B.: Exact theory of two-dimensional self-focusing and one-dimensional self-modulation of waves in nonlinear media. JETP 34(1), 62-69 (1972)

Publisher's Note Springer Nature remains neutral with regard to jurisdictional claims in published maps and institutional affiliations. 